ARtigo de Revisão

REVIEW ARTICLE

\title{
Osteoporose e Gravidez ${ }^{*}$
}

\section{Osteoporosis and Pregnancy}

Francisco Airton Castro da Rocha ${ }^{(1)}$, Francisco Saraiva da Silva Junior ${ }^{(2)}$

\section{RESUMO}

Os autores revisam a literatura sobre os possíveis efeitos da gravidez no desencadeamento de osteoporose, juntamente com uma breve discussão de aspectos da regulação do metabolismo do cálcio na mulher grávida. A carência de estudos a esse respeito é ressaltada. Os autores enfatizam a necessidade de estudos em nossa população, enfocando a possível associação entre paridade e fraturas decorrentes de osteoporose.

Palavras-chave: osteoporose, gravidez e paridade.

\section{INTRODUÇÃO}

Razões de cunho ético limitam os estudos como o intuito de determinar alterações do metabolismo do cálcio e osteoporose durante a gravidez. Adicionalmente, a extrapolação a partir de modelos experimentais é limitada, ante o pequeno período gestacional nos animais de pequeno porte e prole mais numerosa, implicando, como no caso de ratos, a necessidade de rápida e relevante aquisição de massa mineral óssea no esqueleto do feto e na vida pós-natal. Na presente revisão, abordamos alguns aspectos relacionados à fisiologia do metabolismo do cálcio, vitamina $\mathrm{D}$ e hormônio da paratiróide (PTH) na gravidez, incluindo também uma breve revisão de estudos para investigar ocorrência de osteoporose na gravidez. Constatamos a escassez de estudos nessa área. Particularmente em nossa população, não encontramos publicações com casuística relevante sobre a ocorrência de osteoporose na gravidez. Essa realidade reflete um campo inexplorado de pesquisa que pode interessar ao pesquisador em reumatologia no Brasil.

\begin{abstract}
The authors review the literature approaching the possible effects of pregnancy in the development of osteoporosis, coupled to a brief discussion of aspects about the regulation of calcium metabolism in pregnant women. The lack of studies focusing this subject is remarked. The authors emphasize the need for studies based on our population, approaching the possible association of parity and osteoporosis related fractures.
\end{abstract}

Keywords: osteoporosis, pregnancy, parity.

\section{METABOLISMO DO CÁLCIO E GRAVIDEZ}

Presumivelmente, a necessidade de fornecimento de cálcio durante a embriogênese se daria a expensas de uma redução das reservas maternas. Com esse objetivo, ocorreriam alterações metabólicas na mulher grávida e um aporte na amamentação, representando um custo adicional na transferência de cálcio da mãe para a criança. De fato, foi proposto um mecanismo de hiperparatiroidismo secundário, posteriormente não confirmado, que se desenvolveria durante a gravidez, por meio do qual haveria transferência de cálcio das reservas maternas para o feto, podendo causar $o_{\text {osteoporose }}{ }^{(1)}$. Levando em consideração que o pico de massa óssea do indivíduo é alcançado ao redor dos 30 anos de idade, é razoável supor que o atendimento das demandas fetais pode representar um evento importante como fator de risco para um menor pico de massa óssea e, por conseguinte, maior risco de fraturas decorrentes de osteoporose a partir da quinta década de vida da mulher. Apesar dessas conjecturas, considera-se que a perda óssea durante a gra-

\footnotetext{
* Trabalho realizado no Serviço de Reumatologia do Hospital Walter Cantídio. Universidade Federal do Ceará (UFC), Fortaleza, CE, Brasil. Recebido em 02/03/2005. Aprovado, após revisão, em 05/04/2005.

1. Professor adjunto. Disciplina de Reumatologia da Faculdade de Medicina da UFC.

2. Pós-graduando da Disciplina de Reumatologia da Faculdade de Medicina da Universidade de São Paulo (FMUSP), São Paulo, SP, Brasil. Médico Reumatologista do Serviço de Reumatologia do Hospital Walter Cantídio, UFC.

Endereço para correspondência: : Dr. Francisco Airton Castro da Rocha. R. Dr. José Lourenço, 1930, CEP 601 15-281. Fortaleza, CE, Brasil. Tel. (85) 3244-6215; fax (85) 3244-6215; e-mail: arocha@ufc.br
} 
videz e lactação seja mínima e transitória, sendo recuperada após esse período ${ }^{(2)}$.

Curiosamente, não existem dados convincentes na literatura que estabeleçam uma relação direta entre paridade ou anos de amamentação com osteoporose. Embora haja poucos trabalhos relatando menor densidade mineral óssea associada a maior paridade, tempo de amenorréia pós-parto e tempo de amamentação, praticamente inexistem estudos relatando associação direta entre esses últimos eventos e incidência de fraturas relacionadas à osteoporose.

O nascituro tem em média 21 gramas de cálcio no corpo (variação de 13 a 33g), sendo que a maior parte é adquirida (transferida da mãe) durante o último trimestre da gravidez, quando ocorre a fase mais rápida de aquisição de peso pelo feto, durante o seu desenvolvimento intra-uterino ${ }^{(3)}$. A exemplo do que ocorre com outros nutrientes, assim como com o leite materno, a transferência de cálcio da mãe para o feto independe da sua dieta, exceto em situações de muito baixa ingesta, da ordem de menos de 600 gramas de cálcio por $\operatorname{dia}^{(4)}$

Para atender às demandas maternas e fetais, considerando uma ingestão diária ideal de cerca de 1.200 a 1.500 mg, deveriam ocorrer, idealmente, aumento da absorção intestinal e/ou diminuição da excreção urinária de cálcio. Entretanto, caso haja deficit no suprimento nutricional, haverá a mobilização dos estoques maternos de cálcio do esqueleto para atender a demanda fetal, criando-se um mecanismo similar ao hipogonadismo pós-menopausa, imputado como fenômeno importante na fisiopatologia da osteoporose feminina.

Contribuindo para esse incremento no balanço de cálcio, foi relatado aumento nos níveis séricos de 1,25-di-hidroxivitamina D na grávida, por mecanismos ainda não esclarecidos (para revisão, consulte Kovacs) ${ }^{(5)}$. Possivelmente, pelo menos em parte, decorrendo deste aumento, demonstrou-se maior absorção do cálcio pelo intestino, principalmente no terceiro trimestre. Em um estudo em adolescentes grávidas, a fração absorvida de cálcio, medida por espectrometria de massa atômica usando isótopos orais estáveis de cálcio, aumentou em cerca de $60 \%$ a $70 \%$ durante a gravidez, particularmente no terceiro trimestre. No pósparto tardio, por volta de 3 a 4 semanas, independente de estarem amamentando, ocorreu retorno aos valores basais ${ }^{(6)}$. Considerando uma dieta de $1 \mathrm{~g}$ de cálcio por dia, isso representaria um incremento de cerca de $235 \mathrm{mg} /$ dia no terceiro trimestre, o que atenderia a demanda fetal. Entretanto, em razão do aumento na taxa de filtração glomerular nesse período gestacional, associado ao aumento nos níveis séricos de 1,25 di-hidroxivitamina $\mathrm{D}$, como supracitado, ocorre incremento na calciúria, que pode chegar a ser $46 \%$ maior em relação aos níveis da mulher não-grávida ${ }^{(7)}$. Essa combinação de efeitos reduz a eficiência do aumento da absorção de cálcio, possivelmente resultando em perda dos estoques maternos do mineral nesse período.

Com relação aos níveis séricos de PTH, os relatos iniciais foram prejudicados por limitações técnicas na mensuração. Estudos mais recentes revelam que o PTH se mantém na faixa inferior de normalidade, sem grandes variações ao longo da gravidez ${ }^{(5)}$. Esse fato refuta a hipótese de haver um estado de hiperparatiroidismo secundário "fisiológico" durante a gravidez.

O peptídio relacionado ao hormônio da paratiróide (PTHrp) também tem participação controversa no metabolismo do cálcio durante a gravidez. O PTHrp apresenta homologia parcial com o PTH e pode ativar o receptor do PTH. Entretanto, embora no rim e nos ossos o PTHrp possa ter ações similares ao PTH, fragmentos do PTHrp podem apresentar efeitos pleiotrópicos como na regulação da indução do trabalho de parto, suprimindo a contração uterina $^{(8)}$. Há estudos demonstrando elevação dos níveis do PTHrp na gravidez, mas não está clara a relevância clínica desse achado. Isso porque o PTHrp poderia suprimir os níveis séricos de $\mathrm{PTH}$, em face do aumento dos níveis da 1,25-di-hidroxivitamina $\mathrm{D}$ e do cálcio sérico $^{(5)}$. De maneira similar, os níveis de calcitonina sérica também não se alteram significativamente na gravidez, mesmo levando-se em conta a produção extratiroideana, a partir da placenta e do tecido mamário ${ }^{(5)}$.

\section{OCORRÊNCIA DE OSTEOPOROSE NA GRAVIDEZ}

Estudos para determinar alterações do metabolismo do cálcio e da densidade mineral óssea durante a gravidez são importantes para avaliar o seu possível impacto, a longo prazo, no risco de fratura nas mulheres.

Dentre as apresentações clínicas, vale mencionar também a rara ocorrência de osteoporose transitória do quadril associada à gravidez. O quadro clínico é de dor local, marcha antálgica com elevação do quadril ou fratura do quadril ocorrendo no terceiro trimestre. Em recente revisão do assunto, descreveu-se o quadro clínico e alterações obtidas por ressonância nuclear magnética em duas pacientes com osteoporose do quadril na gravidez. As pacientes foram tratadas com calcitonina, sendo uma paciente com início da calcitonina ainda na gravidez e a 
outra no pós-parto, com resolução dos sintomas em 6 a 9 semanas ${ }^{(9)}$. Apesar da raridade desta condição, da qual só se tem relatos de casos, cuja evolução é em geral autolimitada e requer apenas repouso, fraturas femorais têm sido associadas como resultado da osteoporose transitória do quadril na gravidez ${ }^{(10)}$.

Investigações da osteoporose na mulher grávida são limitadas pelo risco de exposição do feto à radiação. A realização de medidas em ossos periféricos é uma estratégia para atenuar esse risco. Outra opção é a utilização da sonometria óssea. A utilização dos chamados marcadores de formação ou de reabsorção óssea, como a dosagem da fosfatase alcalina óssea e osteocalcina séricos, ou dos fragmentos do colágeno tipo I no sangue ou na urina, que já têm grande limitação na sua utilização na população em geral, apresentam maior dificuldade de interpretação na mulher grávida. Isso porque, na gravidez, há que se considerar fatores como: a) variações decorrentes da possível produção dessas substâncias pela placenta; b) sobrecarga volêmica, incidente no último trimestre gestacional com aumento na taxa de filtração glomerular; c) redução no turnover ósseo, em decorrência da menor atividade física.

A maioria dos estudos realizados carece de adequados controles. Em um dos poucos estudos longitudinais realizados, feito em Hong Kong, mulheres de baixo risco obstétrico foram submetidas a três medidas da densidade mineral óssea do calcâneo, realizadas antes de 18 semanas de gestação, entre 28 e 32 semanas e uma terceira medida entre 36 e 38 semanas, utilizando um aparelho de ultrasonometria óssea (Sahara Hologic ${ }^{\circledR}$ ). Avaliando $780 \mathrm{mu}-$ lheres, os autores obtiveram uma média de queda na densidade mineral óssea de $0,040 \mathrm{~g} / \mathrm{cm}^{2}$ entre os trimestres, que alcançou diferença estatisticamente significativa, sendo menor na terceira medida. No mesmo estudo, por regressão logística, evidenciou-se que mulheres com baixa densidade mineral óssea inicial e maior ganho de peso durante a gestação tiveram maior redução da densidade mineral óssea durante a gravidez, ao passo que a doença hipertensiva na gravidez e obesidade prévia não se associaram à perda mineral óssea ${ }^{(11)}$.

Em outro estudo prospectivo, avaliou-se a densidade do osso cortical e trabecular registrados no primeiro e terceiro trimestre de gravidez em 43 mulheres saudáveis, em conjunto com medidas do PTH sérico, calcitonina, osteocalcina e fosfatase alcalina séricos, além do índice de desoxipiridinolina/creatinina urinário. Os resultados mostraram que o osso cortical não se alterou, mas a densidade do osso trabecular apresentou grande variação, sendo identificados dois grupos, a saber: um de perdedoras rápidas (menos de $-3 \%$ ) e outro de perdedoras lentas (mais de $-3 \%$ ), com grande variação interindivíduos. Dentre todos os outros parâmetros avaliados, apenas a osteocalcina sérica foi menor nas perdedoras lentas, quando comparadas às perdedoras rápidas $^{(12)}$. O pequeno número de pacientes e a grande variabilidade nos resultados limitam seriamente estender a interpretação dos achados para a população geral.

Conforme dissemos acima, não há dados determinando de forma clara se a paridade ou a amamentação representam risco importante de fraturas a longo prazo. Um dos poucos estudos desse aspecto, realizado na Turquia, reportou uma associação estatisticamente significativa entre multiparidade, particularmente para mulheres com paridade maior do que cinco filhos, e menor densidade mineral óssea na pós-menopausa, não tendo sido encontrada influência da idade, índice de massa corporal, idades da menarca e menopausa. Entretanto, outros possíveis fatores como tempo de amenorréia pós-parto e tempo de amamentação não foram $\operatorname{avaliados}^{(13)}$.

Os riscos relacionados a antecedentes pessoais, como ser a mulher portadora de artropatias crônicas inflamatórias, tampouco foram mensurados. Seja pela própria fisiopatologia da doença ou pelo tratamento administrado, p.ex. corticosteróides, é possível que haja incremento na perda mineral óssea dessas pacientes, caso engravidem. Adicionalmente, ressaltamos, nas pacientes portadoras de síndrome do anticorpo antifosfolípide, primária ou associada ao lúpus eritematoso sistêmico, o risco adicional, por vezes esquecido, do uso crônico de heparina no surgimento de osteoporose $_{(14)}$.

Recentemente, Pearson et al., estudando uma população do Reino Unido, avaliaram a densidade mineral óssea, de forma prospectiva seqüencial, em 60 mulheres saudáveis e encontraram queda significativa na coluna e no quadril total e trocânter, mas não no colo do fêmur. Após o parto, as mulheres que amamentaram mostraram uma perda maior em todos os sítios mensurados, sendo mais pronunciada a perda na coluna. Aquelas que usaram aleitamento artificial aumentaram ou mantiveram a densidade mineral óssea, enquanto as amamentadoras parciais tiveram resultados intermediários entre os dois outros grupos ${ }^{(15)}$. O pequeno número de pacientes e a carência de controles como índice de massa corporal, sedentarismo e fumo, que sabidamente podem influenciar a massa óssea em mulheres não-grávidas, limitam a interpretação desses dados.

Em um estudo no Japão, não se observou efeito do número de gestações na densidade mineral óssea (DMO), 
realizada em 1.113 mulheres saudáveis, medida sete dias após o parto. Adicionalmente, em 113 mulheres desse mesmo grupo, os autores mediram a DMO após um segundo parto, encontrando níveis maiores que os observados após o primeiro parto. Em uma análise múltipla de regressão logística, não foram encontrados fatores independentes determinantes da DMO, exceto por uma associação negativa entre a idade materna e a DMO no segundo parto $^{(16)}$. Em outro artigo, também um estudo longitudinal, foi medida a DMO por densitometria de dupla emissão de raios X (DEXA) em 46 mulheres antes da concepção e imediatamente após o parto, sendo comparadas à de 30 mulheres que tiveram a DMO medida e não engravidaram. Os resultados mostraram não haver diferença entre os grupos. Durante a gravidez, ocorreu uma pequena redução na DMO em todos os locais, particularmente no trocânter, mas que não alcançou significância estatística ${ }^{(17)}$. O pequeno número de indivíduos analisados certamente influenciou esses resultados e reforça a necessidade de estudos mais completos para que se obtenha dados mais concretos sobre o efeito da gravidez no desencadeamento de osteoporose.

Vale destacar, ainda nesse aspecto, que desconhecemos o efeito nas grávidas de Regiões de alto índice de insolação, como no Norte e Nordeste brasileiros. Baixa exposição aos raios ultravioleta foi associada a níveis séricos menores de 1,25 dihidroxivitamina $\mathrm{D}$ e surgimento de raquitismo nutricional em populações do sul da Argentina. Nessa região, demonstrou-se que a fotoconversão in vitro da próvitamina $D_{3}$ em pré-vitamina $D_{3}$ não se faz durante o inverno em latitudes extremas ao sul da Argentina, quando comparado a Buenos Aires ${ }^{(18)}$. Curiosamente, em um estudo que realizamos em Sobral, CE $\left(3^{\circ} \mathrm{S} / 40^{\circ} \mathrm{E}\right)$, encontramos significativa menor incidência de fraturas atribuíveis à

\section{REFERÊNCIAS}

1. Albright F, Reifenstein EC: Parathyroid Glands and Metabolic Bone Disease. Williams \& Wilkins, Baltimore. 1948.

2. McKenna MJ: Miscellaneous causes of osteoporosis. In: Primer of the metabolic bone diseases and disorders of mineral metabolism. Raven Press, p. 260-1, 1993.

3. Givens MH, Macy IC: The chemical composition of the human fetus. J Biol Chem 102: 7-17, 1933.

4. Koo WW, Walters JC, Esterlitz J, Levine RJ, Bush AJ, Sibai B: Maternal calcium supplementation and fetal bone mineralization. Obstet Gynecol 94: 577-82, 1999.

5. Kovacs CS, Kronenberg HM: Maternal-fetal calcium and bone metabolism during pregnancy, puerperium, and lactation. Endocr Rev 18: 832-72, 1997. osteoporose, quando comparada à observada em populações localizadas em latitudes maiores, sugerindo uma influência, entre outros fatores, do tempo e intensidade de insolação na formação de vitamina D19.

Todos os estudos supracitados foram realizados em populações distintas do Brasil. Revisando a escassa literatura, encontramos um estudo realizado em São Paulo, publicado em 2001, em que se procurou identificar fatores de risco associados a fraturas do fêmur proximal. Foram estudados 73 pacientes, sendo 51 mulheres, todos acima de 65 anos de idade. Os autores relataram associação de baixa atividade física, baixo índice de massa corporal, maior número de gestações e de filhos amamentados com maior risco de fraturas $^{(20)}$. Embora limitado pelo número de pacientes, esse é um estudo, feito em nossa população, que sugere a multiparidade e amamentação como fatores de risco para fraturas osteoporóticas.

Particularmente em relação ao Norte e Nordeste brasileiros, os dados reportados em comunidades brancas ou asiáticas podem não se aplicar. Apenas como exemplo, é possível que numa região com alto e constante índice de insolação, alterações dos níveis de 1,25 dihidroxivitamina D3, como reportados em populações brancas no hemisfério norte, não tenham tanta relevância. Além disso, o papel da miscigenação (negros e índios) na determinação do "osso do brasileiro" ainda está por ser explorado.

Em conclusão, podemos dizer que, aparentemente, a gravidez não parece afetar a densidade mineral óssea de forma permanente. Entretanto, a despeito de poder não alterar a densidade mineral óssea, a literatura carece de estudos sobre o efeito da paridade e amamentação sobre o evento a ser prevenido como decorrência de osteoporose, qual seja, o risco de fraturas.

6. O'Brien KO, Nathanson MS, Mancini J, Witter FR: Calcium absorption is significantly higher in adolescents during pregnancy than in the early postpartum period. Am J Clin Nutr 78: 1188-93, 2003.

7. Kalkwarf HJ, Specker BL: Bone mineral changes during pregnancy and lactation. Endocrine 17: 49-53, 2002.

8. Ferguson II JE, Gorman JV, Bruns DE, et al: Abundant expression of parathyroid hormone related protein in human amnion and its association with labor. Proc Natl Acad Sci USA 89: 8384-8, 1992.

9. Arayssi TK, Tawbi HA, Usta IM, Hourani MH: Calcitonin in the treatment of transient osteoporosis of the hip. Semin Arthritis Rheum 32: 388-97, 2003.

10. Wood ML, Larson CM, Dahners LE: Late presentation of a displaced subcapital fracture of the hip in transient osteoporosis of pregnancy. J Orthop Trauma 17: 582-4, 2003. 
11. To WW, Wong MW, Leung TW: Relationship between bone mineral density changes in pregnancy and maternal and pregnancy characteristics: a longitudinal study. Acta Obstet Gynecol Scand 82: 820-7, 2003.

12. Wisser J, Florio I, Neff M, et al: Changes in bone density and metabolism in pregnancy. Acta Obstet Gynecol Scand 84: 349-54, 2005.

13. Gur A, Nas K, Cevik R, Sarac AJ, Ataoglu S, Karakoc M: Influence of number of pregnancies on bone mineral density in postmenopausal women of different age groups. J Bone Miner Metab 21: 234-41, 2003.

14. Di Munno O, Mazzantini M, Delle Sedie A, Mosca M, Bombardieri $\mathrm{S}$ : Risk factors for osteoporosis in female patients with systemic lupus erythematosus. Lupus 13: 724-30, 2004.

15. Pearson D, Kaur M, San P, Lawson N, Baker P, Hosking D: Recovery of pregnancy mediated bone loss during lactation. Bone 34: 570-8, 2004
16. Matsushita H, Kurabayashi T, Tomita M, Honda A, Takakuwa K, Tanaka K: The effect of multiple pregnancies on lumbar bone mineral density in Japanese women. Calcif Tissue Int 71: 10-3, 2002.

17. Kaur M, Pearson D, Godber I, Lawson N, Baker P, Hosking D: Longitudinal changes in bone mineral density during normal pregnancy. Bone 32: 449-54, 2003.

18. Ladizesky M, Lu Z, Oliveri B, et al: Solar ultraviolet B radiation and photoproduction of vitamin D3 in central and southern areas of Argentina. J Bone Miner Res 10: 545-9, 1995

19. Rocha FAC, Ribeiro AR: Low incidence of hip fractures in an equatorial area. Osteoporosis Int 14: 496-9, 2003.

20. Ramalho AC, Lazaretti-Castro M, Hauache O, et al: Osteoporotic fractures of proximal femur: clinical and epidemiological features in a population of the city of São Paulo. São Paulo Med J 119: 4853,2001 . 